\title{
Aplicabilidad de inteligencia de negocio como soporte en toma de decisiones en la asociación bananera fincas de el oro
}

\section{Applicability of business intelligence as a support for decision making in the banana association fincas de el oro}

\author{
DOI: $10.46932 / \mathrm{sfjdv2n4-048}$
}

Received in: March 1st, 2021

Accepted in: May 30th, 2021

\section{Jazmín Apolo-Romero}

Estudiante de la Carrera Economía Agropecuaria, Universidad Técnica de Machala, Facultad de

Ciencias Agropecuarias, Ecuador.

E-mail: japolo2@utmachala.edu.ec

Víctor Javier Garzón Montealegre

Universidad Técnica de Machala, Facultad de Ciencias Agropecuarias, Ecuador

E-mail: vgarzon@utmachala.edu.ec

Jessica Quezada Campoverde

Universidad Técnica de Machala, Facultad de Ciencias Agropecuarias, Ecuador

E-mail:jquezada@utmachala.edu.ec

\section{Eveligh Prado Carpio}

Universidad Técnica de Machala, Facultad de Ciencias Agropecuarias, Ecuador

E-mail: eprado@utmachala.edu.ec

\section{RESUMEN}

En el presente artículo, el objetivo de esta investigación es indagar la influencia de la inteligencia de negocio para la toma de decisiones en los asociados de la empresa Bananera Fincas de El Oro. Uno de los activos más importantes de la empresa es el manejo de información, esto ha llevado a la proliferación y utilización de software y almacenes de datos. Por esta razón, la Inteligencia de negocio puede generar inteligencia de sus datos, mediante la captura, extracción, filtrado y modelado de la información. Las herramientas tecnológicas para el desarrollo de la inteligencia de negocios han evolucionado satisfactoriamente en los mercados empresariales, empresas agropecuarias a nivel global. En la investigación se aplicó un diseño no experimental y de enfoque mixto. Los cuestionarios aplicados fueron sometidos a un control de calidad, para proceder a codificar y transcribir los datos en formato Excel, donde se utilizó métodos estadísticos para verificar los resultados, mismo fueron exportados y procesados a través del programa SPSS v. 24 aplicando un análisis estadístico inferencial el cual arrojó como resultado que existe una influencia positiva fuerte con un $92.3 \%$ entre la variable inteligencia de negocio y la variable toma de decisiones. Por este motivo es oportuno implementar un Software de inteligencia de negocio en el área de producción de la empresa como instrumento para el análisis de grandes volúmenes de datos. En este sentido el estudio desarrollado en inteligencia de negocio para la toma de decisiones responde a la necesidad planteada desde el análisis de la demanda.

Palabras Clave: Inteligencia de negocio; base de datos; tecnología de la información; regresión. 


\begin{abstract}
In this article, the objective of this research is to investigate the influence of business intelligence for decision making in the associates of the company Bananera Fincas de El Oro. One of the most important assets of the company is the management of information, this has led to the proliferation and use of software and data warehouses. For this reason, business intelligence can generate intelligence from its data, by capturing, extracting, filtering and modeling information. Technological tools for the development of business intelligence have evolved satisfactorily in business markets, agricultural companies at a global level. A non-experimental design with a mixed approach was applied in the research. The questionnaires applied were subjected to a quality control, to proceed to code and transcribe the data in Excel format, where statistical methods were used to verify the results, which were exported and processed through the SPSS v. 24 program applying an inferential statistical analysis which showed as a result that there is a strong positive influence with $92.3 \%$ between the variable Business Intelligence and the variable Decision Making. For this reason it is appropriate to implement a business intelligence software in the production area of the company as an instrument for the analysis of large volumes of data. In this sense, the study developed in business intelligence for decision making responds to the need raised from the demand analysis.
\end{abstract}

Keywords: Business intelligence; database; information technology; regression.

\title{
1 INTRODUCCIÓN
}

La búsqueda del éxito empresarial ha llevado a las organizaciones a buscar estrategias tecnológicas que les permitan adquirir, retener, almacenar y diseminar el conocimiento, de tal manera que les sea posible emplearlo a tiempo y les permita tomar decisiones e innovar en el mercado (D'Arconte, 2018).

Conforme la agroindustria ha ido desarrollando y modernizando, se ha ido encontrando con algunos problemas, uno de ellos la identificación de las estrategias que les permitan incrementar la productividad, en detrimento de la expansión del área agrícola (Nunes et al., 2017), que les ayude a alcanzar el éxito en la empresa.

Un factor muy importante en la actualidad empresarial es la innovación, clave para mejorar los niveles de competitividad de la industria y el respectivo crecimiento económico (Vega de la Cruz et al., 2017), es por esto que se vienen creando nuevos productos y servicios tecnológicos, las cuales afectan de manera positiva en el desempeño organizacional (Matheus et al., 2020). El uso adecuado de la tecnología contribuye a alcanzar los resultados propuestos por la empresa (Kruize et al., 2016).

Por esta razón, en la actualidad las empresas manejan un flujo de información que permite conocer tiempos remotos que se consideraban inimaginables (Rocha \& Mello, 2015). En este contexto, la tecnología está contribuyendo con diversas herramientas que logran un mejor desempeño en el acceso y recopilación datos. 
Una herramienta que ha sido muy utilizada durante las últimas décadas es la inteligencia de negocios, esta permite extraer, explotar, simplificar información y descubrir patrones que se convertirán en futuros eventos de la empresa (Yan et al., 2018).

Algunos trabajos recientes han sido publicados utilizando inteligencia de negocios, en el ámbito empresarial (Wamuyu, 2017); en pequeña y mediana empresas (Verdouw et al., 2015); organización empresarial (Cheng et al., 2020; Jones et al., 2017); agronegocio (Pivoto et al., 2018); Desarrollos para la agricultura en la era digital (Saibene et al., 2020); cultivo de langostino (Peña et al., 2019).

Una alternativa que ayudaría a la mejora del registro de información en el sector bananera en la provincia de El Oro es la aplicación de inteligencia de negocio. Por lo descrito, el objetivo de la presente investigación es analizar la influencia de la inteligencia de negocio sobre la toma de decisiones en la Asociación Agraria Fincas El Oro.

\subsection{INTELIGENCIA DE NEGOCIO EN EL SECTOR EMPRESARIAL}

Una de las principales tendencias sociales ha sido hacia la digitalización, estas han cambiado a la sociedad y los negocios (Tena Medialdea et al., 2018). Las empresas en la actualidad vienen adoptando de forma masiva los sistemas de información empresarial como elemento de apoyo a los procesos de negocios (Tokola et al., 2016). Innovaciones tecnológicas como el internet de las cosas o los sistemas cibernéticos brindan nuevas oportunidades a las empresas (Bach et al., 2018; Yan et al., 2018).

Huang (2018) establece que la inteligencia de negocio puede generar inteligencia de sus datos, mediante la captura, extracción, filtrado y modelado de la información. Las herramientas tecnológicas para el desarrollo de la inteligencia de negocios han evolucionado satisfactoriamente en los mercados empresariales, empresas agropecuarias a nivel global.

En los últimos años, los software para aplicar inteligencia de negocio han surgido, entre ellos Microsoft Power BI, QlikSense, Tableau, Oracle BI, IBM Cognos Analytics, Clear Analytics, Looker, Systum, Zoho Analytics, Yellowfin BI y SAP Business Intelligence (Barros et al., 2015; Guamán, 2019).

Muchas empresas agroindustriales, manufactureras, agropecuarias, etc., cuentan con sistemas comerciales básicos que permiten realizar registros de compras, ventas, producción, facturación. Sin embargo, estos sistemas no son eficientes en la generación de reporte y paneles de control de forma inteligente, es decir, no permiten interactuar con los datos, por lo tanto, se vuelve muy dificultoso para el empresario al momento de tomar decisiones (Merino \& Merino, 2019).

De esta manera, desarrollar inteligencia de negocio en las pymes ecuatorianas permitirá tomar decisiones acertadas y descubrir futuros patrones que servirán en un corto, mediano o largo plazo, para 
estimar futuras ventas, producciones y exportaciones. Es necesario conocer que existen indicadores que permiten medir el presente, pasado y futuro al desarrollar Inteligencia de Negocio (Salinas, 2019).

Algunos investigadores como (Cheng et al., 2020; van Hillegersberg \& Koenen, 2016)señalan que existen algunos constructos que coinciden sobre la relevancia de los modelos de inteligencia de negocios, por medio de estos es posible aprovechar de mejor manera el almacenamiento de grandes volúmenes de información entregados por los sistemas de información gerencial, con el fin de transformar los datos en conocimiento que sirvan para la oportuna toma decisiones (Argüello \& Others, 2015; Salinas, 2019).

\section{METODOLOGÍA}

El presente estudio se desarrolló en la provincia de El Oro, que está ubicada en el litoral sur del Ecuador, centrando la investigación en la Asociación Agraria Bananera Fincas de El Oro.

La investigación realizada se encuentra dentro del paradigma positivista, por lo tanto, con base a este paradigma y la naturaleza de la información recolectada para responder al problema de esta investigación, la misma es de tipo cuantitativa, así mismo, esta investigación busca determinar, evaluar e interpretar las variables relacionadas con el problema, además se intenta encontrar las causas de éste. Esta investigación obedece a un diseño no experimental, de campo, transversal.

\subsection{POBLACIÓN Y MUESTRA}

En la presente investigación se utilizó el muestreo deliberado, crítico o por juicio, la población objeto de estudio estuvo constituida por asociados de la empresa Bananera Fincas de El Oro. Además, se aplicó un cuestionario de 2 dimensiones, 5 indicadores (Tabla 1) acompañado de un total de 20 preguntas dirigidas a los socios, por ser estas personas las que cuentan con los conocimientos necesarios para suministrar la información requerida en la investigación.

Tabla 1. Ficha técnica del cuestionario aplicado.

\begin{tabular}{l|l}
\hline ITEMS & VARIABLES \\
\hline Unidad de Análisis & Asociación Agraria Bananera Fincas El Oro. \\
\hline Técnica & Encuestas aplicadas a asociados o a productores. \\
\hline Tamaño de la muestra & 55 asociados \\
\hline Inteligencia de negocio (ID) & $\begin{array}{l}\text { Sistemas de Información } \\
\text { Tecnologías de Información } \\
\text { Proceso Administrativo }\end{array}$ \\
\hline Toma de decisiones (D) & $\begin{array}{l}\text { Proceso de toma de decisiones } \\
\text { Planeación estratégica. }\end{array}$ \\
\hline
\end{tabular}

ID: Variable independiente; D: Variable dependiente 


\subsection{MÉTODO DE ANÁLISIS DE DATOS}

Los cuestionarios aplicados fueron sometidos a un control de calidad, para proceder a codificar y transcribir los datos en formato Excel, donde se utilizó métodos estadísticos para verificar los resultados obtenidos a través del cuestionario empleando en el programa estadístico IBM SPSS Statistics v. 24. En base a los resultados obtenidos se elaboraron gráficas y tablas para su respectiva interpretación.

De esta manera, la presente investigación se realizó en tres momentos secuenciales en el tiempo. En un primer momento, se utilizará la prueba del alfa de Cronbach para determinar la confiabilidad del instrumento. En un segundo momento, se aplicará una estadística descriptiva que permitirá analizar el comportamiento de las variables mediante los cálculos de porcentajes de participación, distribución de frecuencias, tablas, gráficos. En un tercer momento, posteriormente, se emplea el análisis inferencial con el fin de comprobar las hipótesis que se plantearon, mediante el coeficiente correlación de Pearson, Regresión Lineal y Anova.

Tabla 2. Resumen del procesamiento de los casos y estadísticos de fiabilidad

\begin{tabular}{llllll}
\hline & & N & $\%$ & & \\
\hline \multirow{2}{*}{ Casos } & Válido & 15 & 100 & Alfa de Cronbach & $\mathbf{N}^{\circ}$ de Elementos \\
\hline & Excluidos & 0 & 0 & 0,95 & 20 \\
\hline
\end{tabular}

Según el estadístico de fiabilidad (Alfa de Cronbach) el instrumento conformado por 24 preguntas con una prueba piloto de 15 encuestados no menciona que el valor de Alfa de Cronbach es de 0.86. Es decir, el instrumento tiene una confiabilidad muy alta y por ello nos servirá para poder recolectar los datos, posteriormente.

\subsection{ANÁLISIS DESCRIPTIVO}

Analizando los datos luego de realizar la agrupación por dimensión y variable de estudio, se obtuvo las frecuencias y porcentajes respecto a las respuestas elegidas por los 55 asociados de la empresa Bananera Fincas de El Oro.

Luego de realizar la agrupación de las variables de estudio, se obtuvo las frecuencias y porcentajes respecto a las respuestas elegidas por los 55 asociados de la empresa Bananera Fincas de El Oro. También se planteó hipótesis comprobatorias, coeficiente de correlación de Pearson, Regresión Lineal y Anova, entre variable dependiente (agrupados) e independiente (agrupados), con un nivel de significancia $\alpha<0.05$.

Las hipótesis fueron planteadas en función de: 


\subsection{HIPÓTESIS GENERAL}

Existe relación significativa entre la Inteligencia de negocio y la toma de decisiones de los asociados de la empresa Bananera Fincas de El Oro.

\section{Hipótesis de normalidad:}

Ho: La distribución de la muestra sigue una distribución normal

H1: La distribución de la muestra no sigue una distribución normal.

\section{Correlación de Pearson}

\section{Planteo de Hipótesis de relación}

Ho: $\mathrm{R}=0$; No existe relación significativa entre la Inteligencia de negocio y la toma de decisiones de los asociados de la empresa Bananera Fincas de El Oro.

Ha: $\mathrm{R}>0$; Existe relación significativa entre la Inteligencia de negocio y la toma de decisiones de los asociados de la empresa Bananera Fincas de El Oro.

\section{Ecuación multilineal de la recta regresión}

$\boldsymbol{Y}=\boldsymbol{\beta} \boldsymbol{o}+\boldsymbol{\beta} 1(X 1)+\boldsymbol{\beta} 2(X 2)+\boldsymbol{\beta} 3(X 3)($ Ecuación 2)

\section{Dónde:}

$\mathrm{Y}=\mathrm{VD}=$ Toma de decisiones

$\mathrm{X}=\mathrm{VI}=\boldsymbol{X} \mathbf{1}=$ Sistemas de Información, $\boldsymbol{X} \mathbf{2}=$ Tecnologías de Información, $\boldsymbol{X} \mathbf{3}=$ Proceso Administrativo

De acuerdo a los resultados obtenidos, se puede visualizar mediante una tabla de frecuencia Tabla 3, las 55 personas que están vinculados en la área de producción, contestaron a las preguntas planteadas de la variable independiente "Inteligencia de Negocio", de la siguiente forma: Se evidencia que 3 personas representa el $(5,45 \%)$ establecen que nunca realizan inteligencia de negocio, 12 personas equivalentes a $(21,82 \%)$ respondieron que casi nunca se aplica la Inteligencia de Negocio, 16 miembros representa el $(29,09 \%)$ contestaron indicado que a veces se realiza la Inteligencia de Negocio, mientras que 19 personas equivalentes a $(34,55 \%)$ contestaron que casi siempre se aplica la Inteligencia de Negocio y por último tan solo 5 personas representa el ( 9,09\%) contestaron que siempre se aplica la Inteligencia de Negocio.

Tabla 3. Inteligencia de negocio (variable independiente, datos agrupados)

\begin{tabular}{llll}
\hline & & Frecuencia & Frecuencia Relativa (\%) \\
\hline \multirow{3}{*}{ Válido } & Nunca & 3 & 5,45 \\
& Casi Nunca & 12 & 21,82 \\
& A veces & 16 & 29,09 \\
& Casi Siempre & 19 & 34,55 \\
Total & Siempre & 5 & 9,09 \\
\hline
\end{tabular}


Se puede visualizar mediante la Figura 2, las dimensiones de la variable independiente, al ser analizadas, arrojan valores muy similares estableciendo que casi siempre aplican Sistema de información (29,09\%), Tecnologías de la información $(43,64 \%)$ y Proceso administrativo $(34,55 \%)$ en la empresa Bananera Fincas de El Oro, sin embargo, esto es un panorama favorable para aplicar a futuro la inteligencia de negocio.

Figura 1. Dimensiones a) Sistema de información. b) Tecnologías de la información. c) Proceso administrativo.
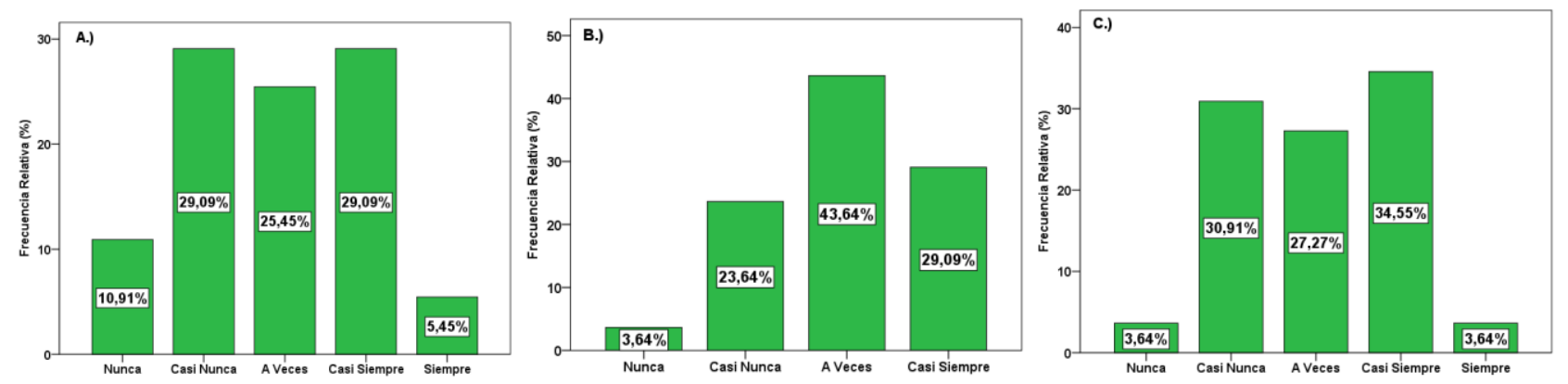

Los resultados de la variable dependiente "Toma de decisiones" se las puede apreciar en la tabla de frecuencias Tabla 4, las 55 personas que son parte de la empresa Bananera Fincas de El Oro contestaron las preguntas planteadas de la siguiente forma: Se evidencia que 6 personas representan el $(10,91 \%)$ indicando que nunca realizan toma de decisiones, 12 personas equivalentes a $(21,82 \%)$ establecen que casi nunca se aplica la toma de decisiones, 15 miembros representa el $(27,27 \%)$ respondieron indicado que a veces se realiza la toma de decisiones, 18 personas representa el (32.73\%) contestaron que casi siempre se aplica la toma de decisiones y finalmente tan solo 4 personas representa el $(7,27 \%)$ contestaron que siempre se aplica la toma de decisiones.

Tabla 4. Toma de decisiones (variable dependiente, datos agrupados)

\begin{tabular}{llll}
\hline & & Frecuencia & Frecuencia Relativa $(\%)$ \\
\hline & Nunca & 6 & 10,91 \\
Válido & Casi Nunca & 12 & 21,82 \\
& A veces & 15 & 27,27 \\
& Casi Siempre & 18 & 32,73 \\
\hline & Siempre & 4 & 7,27 \\
\hline
\end{tabular}

Al observar las dos dimensiones de la variable dependiente, según los resultados mostrados en la Figura 4, estos indican que el área de producción aplica a veces el proceso de toma de decisiones $(50,91 \%)$ y planeación estratégica (34,55\%), mientras que se denotan valores muy similares en casi siempre, en ambas dimensiones, y para finalizar, los demás escenarios existen variabilidad porcentual pero muy baja, esto según la encuesta planteada. 


\section{SOUTH FLORIDA}

JOURNAL OF DEVELOPMENT

Figura 2. Dimensiones d) Proceso de toma de decisiones. e) Planificación estratégica
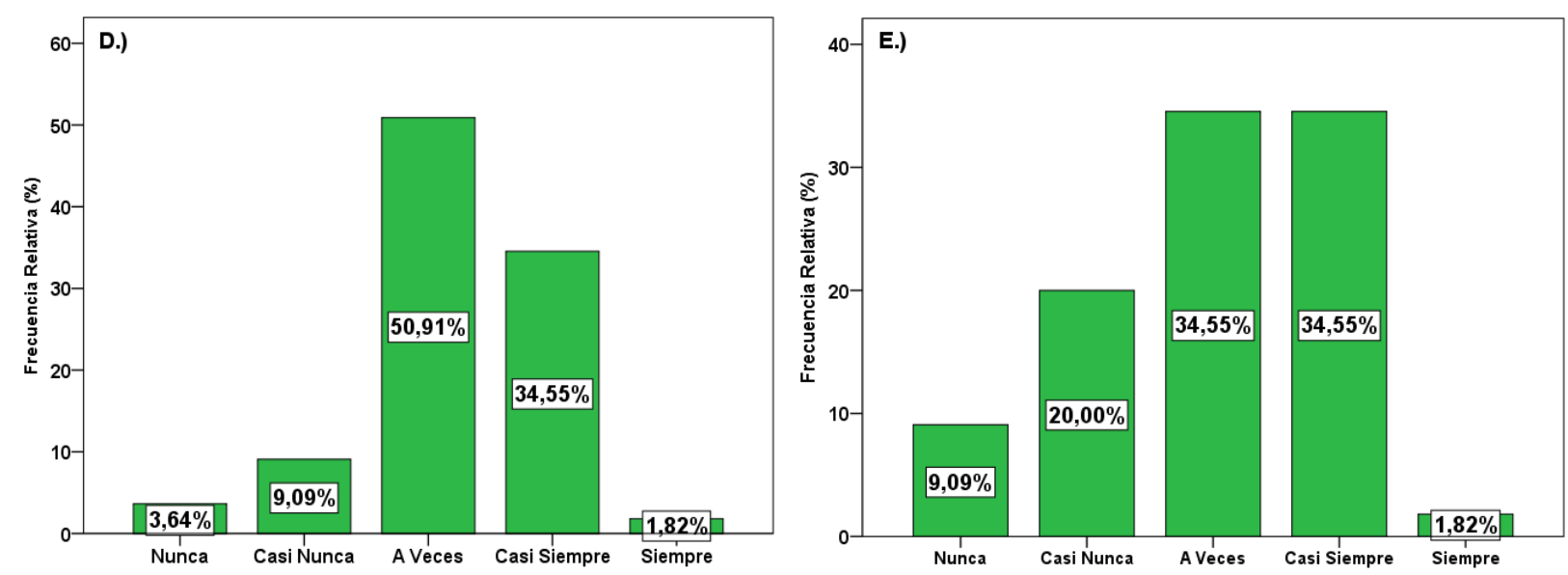

De acuerdo al resultado de la prueba de normalidad mediante el test de Kolmogorov Smirnov (Tabla 5) que se verificó que la variable Inteligencia de negocio y la variable Toma de decisiones tienen un nivel de significancia mayor al nivel de significancia asumida $(0.63 ; 0.200>0.05)$. Por lo tanto, se acepta la Ho y se rechaza la H1, por lo cual la distribución de los datos recolectados tiene una distribución normal y se deberá emplear pruebas paramétricas.

Tabla 5. La prueba de Kolmogorov-Smirnov

\begin{tabular}{llll}
\hline & & & \\
& & $\begin{array}{c}\text { Inteligencia de negocio } \\
\text { (Agrupados) }\end{array}$ & $\begin{array}{c}\text { Toma de decisiones } \\
\text { (Agrupados) }\end{array}$ \\
& & & 55 \\
\hline $\mathbf{N}$ & & 55 & 31,07 \\
Parámetros normales a*b & Medias & 30,13 & 8,364 \\
& Desviación Típica & 8,888 & 0,101 \\
Diferencias más extremas & Absoluta & 0,116 & 0,630 \\
Z de Kolmogorov-Smirnov & Positiva & 0,116 & $-0,101$ \\
Sig. Asintót. (bilateral) & Negativa & $-0,089$ & $\mathbf{0 , 1 0 1}$ \\
\hline
\end{tabular}

\subsection{ANÁLISIS INFERENCIAL}

La contrastación de las hipótesis planteadas se realizó mediante la comprobación de existencia de correlación entre las variables de estudio, la prueba de resumen de Modelo, el Anova y los coeficientes.

Como podemos observar en la Tabla 6, la correlación es de 0.957 que de acuerdo a los niveles de correlación existe una correlación positiva considerable; Así mismo se observa que la significancia encontrada es 0.0001 que es menor al nivel de significancia de $0.05,(0,0001<0,05 ; 95 \% ; Z=1.96)$. Por lo 
tanto, se rechaza la hipótesis nula y se acepta la hipótesis alterna: existe relación significativa entre la inteligencia de negocio y la toma de decisiones de los asociados de la empresa Bananera Fincas de El Oro.

Tabla 6. La correlación de Pearson para la hipótesis general

\begin{tabular}{|c|c|c|c|c|}
\hline & & & $\begin{array}{l}\text { Inteligencia de negocio } \\
\text { (Agrupados) }\end{array}$ & $\begin{array}{l}\text { Toma de decisiones } \\
\text { (Agrupados) }\end{array}$ \\
\hline \multirow{2}{*}{$\begin{array}{l}\text { Inteligencia de negocio } \\
\text { (Agrupados) }\end{array}$} & $\begin{array}{l}\text { Correlación de Pearson } \\
\text { (bilateral) }\end{array}$ & Sig & Asintót.1,00 & $\begin{array}{l}0,957 \\
0,000\end{array}$ \\
\hline & $\mathrm{N}$ & & 55 & 55 \\
\hline \multirow{2}{*}{$\begin{array}{l}\text { Toma de } \\
\text { (Agrupados) }\end{array}$} & $\begin{array}{l}\text { Correlación de Pearson } \\
\text { (bilateral) }\end{array}$ & Sig & $\begin{array}{c}\text { Asintót.0,957** } \\
0,000 \\
\end{array}$ & 1,00 \\
\hline & $\mathrm{N}$ & & 55 & 55 \\
\hline
\end{tabular}

\subsection{PRUEBA DE REGRESIÓN LINEAL}

Observando la Tabla 7 se tiene que el coeficiente de Pearson conjunta es $\mathrm{R}=0,963$. Así mismo el $\mathrm{R}^{2}$ corregido $=0,923$, dicho valor se interpreta según la Tabla 7 de porcentaje estandarizado de Martín (2017) donde la variable toma de decisiones tiene una tendencia muy alta en cambiar en un $92.3 \%$ por acción de la variable inteligencia de negocio y sus dimensiones, la cual es significativa.

Tabla 7. Resumen del Modelo de Hipótesis General

\begin{tabular}{|c|c|c|c|c|c|c|c|}
\hline \multicolumn{8}{|c|}{ Estadísticos de cambios } \\
\hline Modelo & $\mathrm{R}$ & $\mathrm{R}$ cuadrado & $\begin{array}{ll}\mathrm{R} \quad \text { cuadrado } & \text { Error } \\
\text { Estándar de la } & \text { Cambio en } \\
\text { ajustado } & \text { estimación cuadrado }\end{array}$ & $\begin{array}{l}\text { RCambio } \\
\text { en F }\end{array}$ & gl1 & $\mathrm{gl} 2$ & $\begin{array}{l}\text { Sig. Cambio } \\
\text { en F }\end{array}$ \\
\hline 1 & $0,963^{\mathrm{a}}$ & 0,928 & 2,317 & 217,527 & 3 & 51 & 0,000 \\
\hline
\end{tabular}

a. Predictores: (Constante), Sistemas de Información, Tecnologías de Información, Proceso Administrativo.

b. Variable dependiente: Toma de decisiones

El análisis de ANOVA Tabla 8, se observa que el valor "sig." es de 0,0001 que es menor a 0,05 el nivel de significancia, entonces se rechaza la hipótesis nula (Ho), por lo tanto, se acepta la Hipótesis alterna (Ha), y se afirma con un nivel de confianza del 95\%, que la inteligencia de negocio influye significativamente en la toma de decisiones del área de producción.

Tabla 8. Hipótesis general ANOVA

\begin{tabular}{llllll}
\hline Modelo & Suma de cuadrados & gl & Media cuadrática & F & Sig \\
\hline Regresión & 3503,877 & 3 & 1167,959 & 217,527 & $0,000^{\mathrm{b}}$ \\
Residual & 273,832 & 51 & 5,369 & & \\
Total & 3777,709 & 54 & & & \\
\hline
\end{tabular}

a. Variable dependiente: Toma de decisiones

b. Predictores: (Constante), Sistemas de Información, Tecnologías de Información, Proceso Administrativo. 
En la Tabla 9, el $\boldsymbol{\beta} \boldsymbol{o}=2.040$; es el punto de intersección de la recta con el eje vertical del sistema de coordenadas, El coeficiente no estandarizados B muestra que la dimensión que más influye en la variable Toma de decisiones es el Tecnologías de Información $(1,237)$ luego los Proceso Administrativo $(1,162)$ y finalmente Sistemas de Información $(0,571)$. Los valores de la "sig." 0.000, 0.000 y 0.001 de las dimensiones Proceso Administrativo, Sistemas de Información y Tecnologías de Información; respectivamente son menores a 0.05 el nivel de significancia por lo tanto se afirma con un $95 \%$ de confianza que las tres dimensiones influyen significativamente en la Toma de decisiones del área de producción.

Tabla 9. Coeficientes de hipótesis generales

\begin{tabular}{|c|c|c|c|c|c|}
\hline \multirow[b]{2}{*}{ Modelo } & \multicolumn{2}{|c|}{ Coeficientes no estandarizados } & \multicolumn{2}{|c|}{$\begin{array}{l}\text { Coeficientes } \\
\text { estandarizados }\end{array}$} & \multirow[b]{2}{*}{ Sig. } \\
\hline & B & Error típ. & Beta & $\mathbf{t}$ & \\
\hline (Constante) & 2,040 & 1,301 & & 1,568 & 0,123 \\
\hline Sistema de información & 0,571 & 0,169 & 0,299 & 3,379 & 0,001 \\
\hline Tecnologías de información & 1,237 & 0,179 & 0,352 & 6,919 & 0,000 \\
\hline Proceso administrativo & 1,162 & 0,272 & 0,418 & 4,272 & 0,000 \\
\hline
\end{tabular}

a. Variable dependiente: Toma de decisiones (agrupado)

La variable Toma de decisiones está cambiando en 57,1\% cuando la variable Sistemas de Información se incrementa en una unidad, cambia en un $123.7 \%$ cuando la variable Tecnologías de Información se incrementa en una unidad y cambia en un 116.2\% cuando la variable Proceso Administrativo se incrementa en una unidad.

\section{DISCUSIÓN}

El objetivo del presente estudio fue indagar la influencia de la inteligencia de negocio sobre la toma de decisiones de los asociados de la empresa Bananera Fincas de El Oro; de acuerdo a los resultados obtenidos se ha determinado que existe una influencia muy alta de la variable independiente Inteligencia de Negocio sobre la variable dependiente Toma de Decisiones. Estos resultados coinciden con lo mencionado por Huang (2018) afirma que los Sistemas de Información en el negocio, las personas y las organizaciones buscan utilizar la información de manera específica para tomar decisiones sólidas y para resolver problemas, estas dos prácticas relacionadas son la base de toda empresa exitosa.

De acuerdo a la prueba de hipótesis general se observa que, como resultado de la prueba de $\mathrm{R}$ de Pearson, existe una correlación significativa de 0.963 con un valor calculado para $\mathrm{p}=0.000$, lo que indica que la correlación es positiva muy fuerte. Adicionalmente se observa en la Tabla 7, que la variación de la Toma de decisiones del área de producción, está explicada por una fuerza de 92.3\% de la Inteligencia de 
Negocio. Por lo tanto, se toma la decisión de rechazar la hipótesis nula y se acepta la hipótesis alterna, dando como conclusión que existe una influencia muy alta de la Inteligencia de Negocio sobre la Toma de decisiones de la empresa.

Por este motivo es oportuno implementar un Software de inteligencia de negocio como instrumento para el análisis de volúmenes de datos coincidiendo con lo planteado por Cheng et al., (2020); van Hillegersberg \& Koenen, (2018); Salinas (2019). En este sentido el estudio desarrollado en inteligencia de negocio en la toma de decisiones en la asociación bananera Fincas El Oro responde a la necesidad planteada desde el análisis de la demanda.

\section{CONCLUSIONES Y TRABAJOS FUTUROS}

Algunas dimensiones como el proceso administrativo, sistemas de información y tecnologías de información, tienen influencia sobre el desempeño de los diferentes sectores productivos, es por esto que algunas organizaciones ya están utilizando la inteligencia de negocios, el sector de recolección de concha no es la excepción, es por esto que en la actualidad muchas organizaciones ya están implementando este tipo de herramientas para mejorar su desempeño y competitividad en el mercado.

La inteligencia de negocios como herramienta de gestión empresarial sirve de utilidad para el análisis de un gran volumen de datos, de esta manera será posible disponer de la información oportuna con la finalidad de diseñar estrategias para la organización, que sean adecuadas y sostenibles.

Como se puede evidenciar en los resultados obtenidos en la investigación, el uso de la inteligencia de negocios, si se implementa en la empresa Bananera Fincas de El Oro, puede ser de mucha utilidad al momento de tomar decisiones, lo cual a su vez puede mejorar los resultados que obtengan en la producción bananera, incluso pueden tener una mejor planificación en cuanto a los volúmenes a recolectar, pudiendo comercializar y exportar el producto de manera más segura.

Existe una muy alta influencia de la variable independiente inteligencia de negocio sobre la variable dependiente toma de decisiones.

Los trabajos futuros se centran en la aplicabilidad de inteligencia de negocio en la producción y comercialización de arroz (Oryza Sativa). Gestión en la Cadena de valor utilizando visualizaciones mediante el programa Microsoft Power BI. 


\section{REFERENCIAS CONSULTADAS}

Argüello, A., \& Others. (2015). Agroecology: scientific and technological challenges for agriculture in the 21st century in Latin America. Agronomia Colombiana, 33(3), 391-398. http://www.scielo.org.co/scielo.php?script=sci_arttext\&pid=S0120-99652015000300013

Bach, M. P., Jaklič, J., \& Vugec, D. S. (2018). Understanding impact of business intelligence to organizational performance using cluster analysis: does culture matter. International Journal of $\begin{array}{lllll}\text { Information Systems } & \text { Mandect }\end{array}$ https://aisel.aisnet.org/cgi/viewcontent.cgi?article=1040\&context=ijispm

Barros, V. F. de A., Ramos, I., \& Perez, G. (2015). Information systems and organizational memory: a literature review. Jistem - Journal of Information Systems and Technology Management, 12(1), 45-63. https://doi.org/10.4301/S1807-17752015000100003

Cheng, C., Zhong, H., \& Cao, L. (2020). Facilitating speed of internationalization: The roles of business intelligence and organizational agility. Journal of Business Research, 110, 95-103. https://doi.org/10.1016/j.jbusres.2020.01.003

D’Arconte, C. (2018). Business Intelligence applied in Small Size for Profit Companies. Procedia Computer Science, 131, 45-57. https://doi.org/10.1016/j.procs.2018.04.184

Guamán, M. A. A. (2019). La usabilidad en los sistemas de inteligencia de negocios, un caso práctico. Ciencia Digital, 3(3.3), 319-330. https://doi.org/10.33262/cienciadigital.v3i3.3.824

Huang, Y., Chen, Z.-X., Yu, T., Huang, X.-Z., \& Gu, X.-F. (2018). Agricultural remote sensing big data: Management and applications. Journal of Integrative Agriculture, 17(9), 1915-1931. https://doi.org/10.1016/S2095-3119(17)61859-8

Jones, J. W., Antle, J. M., Basso, B., Boote, K. J., Conant, R. T., Foster, I., Godfray, H. C. J., Herrero, M., Howitt, R. E., Janssen, S., Keating, B. A., Munoz-Carpena, R., Porter, C. H., Rosenzweig, C., \& Wheeler, T. R. (2017). Brief history of agricultural systems modeling. Agricultural Systems, 155, 240-254. https://doi.org/10.1016/j.agsy.2016.05.014

Kruize, J. W., Wolfert, J., Scholten, H., Verdouw, C. N., Kassahun, A., \& Beulens, A. J. M. (2016). A reference architecture for Farm Software Ecosystems. Computers and Electronics in Agriculture, 125, 12-28. https://doi.org/10.1016/j.compag.2016.04.011

Matheus, R., Janssen, M., \& Maheshwari, D. (2020). Data science empowering the public: Data-driven dashboards for transparent and accountable decision-making in smart cities. Government Information Quarterly, 37(3), 101284. https://doi.org/10.1016/j.giq.2018.01.006

Merino, E. M. G., \& Merino, M. J. G. (2019). Análisis de los Modelos de Inteligencia de Negocios basados en Big Data en las Pymes del Ecuador. Revista Científica Ciencia y tecnología, 18(18). http://cienciaytecnologia.uteg.edu.ec/revista/index.php/cienciaytecnologia/article/view/436

Nunes, A. L. P., Abi-Saab, O. J. G., \& Ralisch, R. (2017). Customer relationship management in the agricultural machinery market/Customer relationship management no setor de maquinas agricolas. Ciencia Rural, 
https://go.gale.com/ps/i.do?id=GALE\%7CA502000360\&sid=googleScholar\&v=2.1\&it=r\&linkaccess=a bs\&issn $=01038478 \& \mathrm{p}=\mathrm{AONE} \& \mathrm{sw}=\mathrm{w}$

Peña, C., Risco, G., Cardoza, L., Ubillus, E., \& Olaya, C. (2019). Modelo bioeconómico que pronostica las consecuencias técnicas, biológicas y económicas en el cultivo de langostino. Manglar, 16(1), 3-10. https://doi.org/10.17268/manglar.2019.002

Pivoto, D., Waquil, P. D., Talamini, E., Finocchio, C. P. S., Dalla Corte, V. F., \& de Vargas Mores, G. (2018). Scientific development of smart farming technologies and their application in Brazil. Information Processing in Agriculture, 5(1), 21-32. https://doi.org/10.1016/j.inpa.2017.12.002

Rocha, G. V., \& Mello, C. H. P. (2015). How to develop technology roadmaps? The case of a Hospital Automation Company. Production, 26(2), 345-358. https://doi.org/10.1590/0103-6513.173614

Saibene, Y. B., Caldera, J., \& Ramos, L. (2020). Cosechando Datos. Desarrollos para la agricultura en la era digital. Electronic Journal of SADIO (EJS), 19(1), 64-95. https://www.45jaiio.sadio.org.ar/index.php/EJS/article/view/158

Salinas, e. W. H. (2019). Inteligencia de negocios aplicada a la gestión de ventas de una empresa agroindustrial. 7(2), 33-38. https://doi.org/10.18050/cientifi-k.v7i2.2140

Tena Medialdea, J., Prieto Ruiz, J. A., Fagoaga García, C., Calvo Capilla, A., Chirivella Martorell, J., \& Bueso Rodenas, J. (2018). Potential of science to address the hunger issue: Ecology, biotechnology, cattle breeding and the large pantry of the sea. Journal of Innovation \& Knowledge, 3(2), 82-89. https://doi.org/10.1016/j.jik.2017.12.007

Tokola, H., Gröger, C., Järvenpää, E., \& Niemi, E. (2016). Designing Manufacturing Dashboards on the Basis of a Key Performance Indicator Survey. Procedia CIRP, 57, 619-624. https://doi.org/10.1016/j.procir.2016.11.107

van Hillegersberg, J., \& Koenen, S. (2016). Adoption of web-based group decision support systems: experiences from the field and future developments. International Journal of Information Systems and Project Management, 4(1),

https://aisel.aisnet.org/cgi/viewcontent.cgi?article=1070\&context=ijispm

49-64.

Vega de la Cruz, L. O., Lao León, Y. O., \& Julbe, A. F. N. (2017). Proposal of a coefficient to evaluate the management of the internal control. Contaduría $Y$ Administración, 62(2), 699-714. https://doi.org/10.1016/j.cya.2017.03.003

Verdouw, C. N., Robbemond, R. M., \& Wolfert, J. (2015). ERP in agriculture: Lessons learned from the Dutch horticulture. Computers and Electronics in Agriculture, 114, 125-133. https://doi.org/10.1016/j.compag.2015.04.002

Wamuyu, P. K. (2017). Use of cloud Computing Services in micro and Small Enterprises: A fit perspective. http://erepo.usiu.ac.ke/handle/11732/4336

Yan, M., Liu, P., Wen, F., Zhang, C., Zhao, R., Chen, W., Liu, X., \& Liu, Y. (2018). Research on precision management of farming season based on big data. EURASIP Journal on Wireless Communications and Networking, 2018(1), 1-10. https://doi.org/10.1186/s13638-018-1161-y 